\title{
Efficacy of rifabutin-based triple therapy as second-line treatment to eradicate helicobacter pylori infection
} José M Navarro-Jarabo*1, Nuria Fernández ${ }^{1}$, Francisca L Sousa², Encarnación Cabrera ${ }^{3}$, Manuel Castro ${ }^{4}$, Luz M Ramírez ${ }^{5}$, Robin Rivera ${ }^{1}$, Esther Ubiña ${ }^{1}$, Francisco Vera ${ }^{1}$, Isabel Méndez ${ }^{1}$, Francisco Rivas-Ruiz ${ }^{6}$, José L Moreno ${ }^{7}$ and Emilio Perea-Milla ${ }^{6}$

Address: ${ }^{1}$ Unidad de Aparato Digestivo, Hospital Costa del Sol, Ctra Nacional 340, km 187, 29600 Marbella, Spain, ${ }^{2}$ Sección de Aparato Digestivo, Hospital de Motril, Av. Enrique Martín Cuevas, S/N 18600 Motril, Spain, ${ }^{3}$ Servicio de Aparato Digestivo, Hospital General de Especialidades de Jaén, Avda. del Ejército Español, 10. 23007 Jaén, Spain, ${ }^{4}$ Servicio de Aparato Digestivo, Hospital Universitario Ntra. Sra. de Valme, Ctra. de Cádiz km. 548,9, 41014 Sevilla, Spain, 5 Sección de Aparato Digestivo, Hospital Comarcal Valle de los Pedroches, Juan del Rey Calero s/n, 14400 Pozoblanco, Córdoba, Spain, 6 Unidad de Apoyo a la Investigación, Hospital Costa del Sol, Ctra Nacional 340, km 187, 29600 Marbella, Spain and ${ }^{7}$ Unidad de Farmacia, Hospital Costa del Sol, Ctra Nacional 340, km 187, 29600 Marbella, Spain

Email: José M Navarro-Jarabo* - jnavarro@hcs.es; Nuria Fernández - jnavarro@hcs.es; Francisca L Sousa - jarabo@tiscali.es; Encarnación Cabrera - jarabo@tiscali.es; Manuel Castro - jarabo@tiscali.es; Luz M Ramírez - lmramirez@yahoo.es; Robin Rivera - robinriverai@yahoo.es; Esther Ubiña - estherillaua@hotmail.com; Francisco Vera - jarabo@tiscali.es;

Isabel Méndez - chabeli_estepona@hotmail.com; Francisco Rivas-Ruiz - frivasr@hcs.es; José L Moreno - jlmharo@hcs.es; Emilio PereaMilla - eperea@hcs.es

* Corresponding author

Published: 25 July 2007

BMC Gastroenterology 2007, 7:31 doi:|0.|I86/|47|-230X-7-31
Received: 22 December 2006

Accepted: 25 July 2007

This article is available from: http://www.biomedcentral.com/I47I-230X/7/3I

(c) 2007 Navarro-Jarabo et al; licensee BioMed Central Ltd.

This is an Open Access article distributed under the terms of the Creative Commons Attribution License (http://creativecommons.org/licenses/by/2.0), which permits unrestricted use, distribution, and reproduction in any medium, provided the original work is properly cited.

\begin{abstract}
Background: Rifabutin has been found to be effective in multi-resistant patients after various treatment cycles for Helicobacter pylori (HP) infection, but it has not been analysed as a second-line treatment. Therefore, we seek to compare the effectiveness of a treatment regimen including rifabutin versus conventional quadruple therapy (QT).

Methods: Open clinical trial, randomised and multi-centre, of two treatment protocols: A) Conventional regime -QT(omeprazole $20 \mathrm{mg}$ bid, bismuth citrate $120 \mathrm{mg}$ qid, tetracycline $500 \mathrm{mg}$ qid and metronidazole $500 \mathrm{mg}$ tid); B) Experimental one -OAR- (omeprazole $20 \mathrm{mg}$ bid, amoxicillin I gr bid, and rifabutin $150 \mathrm{mg}$ bid), both taken orally for 7 days, in patients with HP infection for whom first-line treatment had failed. Eradication was determined by Urea Breath Test (UBT). Safety was determined by the adverse events.

Results: 99 patients were randomised, QT, $n=54$; OAR, $n=45$. The two groups were homogeneous. In 8 cases, treatment was suspended (6 in QT and 2 in OAR). The eradication achieved, analysed by ITT, was for QT, 38 cases (70.4\%), and for OAR, 20 cases (44.4\%); $\mathrm{P}=0.009$, OR = I.58. Of the cases analysed PP, QT were $77.1 \%$; OAR, $46.5 \%$; $P=0.002$. Adverse effects were described in $64 \%$ of the $Q T$ patients and in $44 \%$ of the OAR patients $(P=0.04)$.

Conclusion: A 7-day rifabutin-based triple therapy associated to amoxicillin and omeprazole at standard dose was not found to be effective as a second-line rescue therapy. The problem with quadruple therapy lies in the adverse side effects it provokes. We believe the search should continue for alternatives that are more comfortably administered and that are at least as effective, but with fewer adverse side effects.
\end{abstract}

Trial Registration: Current Controlled Trials ISRCTN8I058036 


\section{Background}

Helicobacter pylori infection plays an important role in the phatogenesis of chronic gastritis, gastroduodenal ulcer, maltoma and gastric adenocarcinoma. First-line treatment with triple therapy (proton pump inhibitor (PPI)- associated with clarithromycin and amoxicillin or metronidazole) is widely accepted and applied $[1,2]$ and has an effectiveness rate of almost $80 \%$. Nevertheless, a considerable proportion of patients fail to respond to the latter treatment, and for these there is no ideal treatment $[3,4]$. Quadruptherapy (PPI - associated with bismuth citrate, tetracycline and metronidazole) is the most commonly used second-line treatment, with an eradication rate of 57-95\% [4-7]. However, this is not totally satisfactory, due to the complexity of the dosing regimen and the frequency of associated side effects [8]. Thus, alternative options to be applied must be more effective, simpler and better tolerated than the quadruple therapy.

In recent years, it has been shown that some antibiotics can be useful in such circumstances. One of these is rifabutin: derived from rifampicin, it is used as a rescue treatment against mycobacterium tuberculosis and as a prophylactic against the mycobacterium avium intracellulare infection of HIV-positive patients. It has been shown to be effective in eradicating HP $[9,10]$. In relation to this latter effect, it is very effective in vitro [11-13], achieving lower levels of minimum inhibitory concentration than obtained by clarithromycin and amoxicillin [14]. Moreover, its effectiveness does not depend on the $\mathrm{pH}$ of the medium [15].

Many features make rifabutin-based therapy an interesting alternative for clinical application to achieve the eradication of HP. Firstly, it is effective at low doses [16] (300 $\mathrm{mg}$ ), and so its side effects are minimised [17]. Secondly, the primary resistance of HP to rifabutin in the population at large is non-existent, because this antibiotic is only used for very specific clinical situations $[11,18]$. Finally, its efficacy is not reduced by the resistance that HP may develop to other antibiotics, especially clarithromycin and metronidazole $[16,18,26]$, and so it can be administered as a rescue treatment without the need for a prior antibiogram.

Rifabutin-based therapy has been applied as a rescue treatment for patients for whom one or more other treatments have failed, i.e. as a rescue regimen for difficult to treat patients $[16,10,19,20,26]$. No study of it has been made as a second-line treatment, among a homogeneous group of patients for whom a single eradication treatment of triple therapy with IBP, clarithromycin and amoxicillin/metronidazole has failed.
To examine the hypothesis that a regimen including rifabutin may be as effective as one based on quadruple therapy as a rescue treatment, and moreover, that it may achieve higher levels of clinical tolerance, we designed a multicentre, randomised, open clinical trial, with the participation of five hospitals in southern Spain.

\section{Methods \\ Study Design}

This study was performed in acordance with the declaration of Helsinki, and was approved by the Clinical Trials Committee of the Autonomous Administration of Andalucía, and by the corresponding committee of Hospital Costa del Sol (Marbella), Hospital de Motril (Granada), Hospital de Especialidades (Jaen), Hospital de Valme (Sevilla) and Hospital Valle de los Pedroches (Pozoblanco).

Patients were allocated to one of the following treatment regimens: A) OAR (Omeprazole $20 \mathrm{mg} / 12 \mathrm{~h}$, Amoxicillin $1 \mathrm{gr} / 12 \mathrm{~h}$, Rifabutin $150 \mathrm{mg} / 12 \mathrm{~h}$ ); and B) QT (Omeprazole $20 \mathrm{mg} / 12 \mathrm{~h}$, Bismuth Citrate $120 \mathrm{mg} / 6 \mathrm{~h}$, Tetracycline $500 \mathrm{mg} / 6 \mathrm{~h}$, and Metronidazole $500 \mathrm{mg} / 8 \mathrm{~h}$ ). In both cases, the medicaments were administered orally, for 7 days. Patients were progressively included in the trial as determined by the application of a random number table, with an entry number that was unknown to both researchers and patients. All patients signed a written informed consent form. The medication provided was not masked, because the main aim of the trial is influenced by adherence to the treatment regimen, which in turn is affected by the ease, or otherwise, with which the treatment is administered. The medication was prepared by the pharmaceutical service of the organising healthcare provider, delivered in a sealed package, with the number of capsules necessary for each group, and distributed to each participating hospital. Each patient included in the study received a clinical examination (or replied to a telephone interview) to identify the side effects caused by the treatment, following a standardised protocol [21]. At 45 days after ending the treatment, a secondary medical review was performed, at which eradication of HP was determined by UBT and, again, side effects were identified. At this moment, surplus medication was returned. Consumption by the patient of less than $90 \%$ of the capsules initially provided was evaluated as non-fulfilment of the recommended therapy. The patient was classified as having withdrawn from the study if follow up was not performed.

\section{Inclusion and exclusion criteria}

Patients in whom Helicobacter pylori infection persisted after a triple therapy treatment were included. Persistence of infection was determined by a breath test, by a rapid urease test, by pathological anatomy or by culture. The initial diagnosis of Helicobacter pylori infection was 
made by invasive methods (rapid ureasa test and/or pathological anatomy and/or Helicobacter culture) or by noninvasive ones, i.e. a urea breath test decided upon after infrared spectrophotometry or mass-spectrometry. All the patients were given an initial endoscopy prior to the first attempt at eradicating the infection. Patients were excluded from the trial if they withheld consent, if they had initially been treated by the "Test and Treat" procedure or if a baseline endoscopy was not obtained. Also excluded were those patients for whom fulfilment of the treatment regimen and attendance at follow-up appointments could not reasonably be expected. Other conditions for exclusion were HIV positive status, active alcoholism, addiction to drugs, age less than 18 years or more than 75 years, the suspicion of tuberculous infection, either because of a positive intradermal reaction to Mantoux and compatible thorax radiography, or if the patient had previously received tuberculostatic treatment, or a known allergy to any of the components of either of the two treatment regimens. Patients who had received quadruple therapy as first-line treatment, or any other treatment including bismuth (e.g., ranitidine bismuth citrate), or antibiotics during the previous month, were excluded from the trial. In addition, patients with severe associated diseases (cardiac insufficiency, respiratory insufficiency, chronic kidney insufficiency, hepatic insufficiency, advanced neoplasic diseases), as well as those who were pregnant or lactating, were excluded.

\section{Principal variable}

Eradication was defined as having occurred when a negative UBT result was recorded, with urea marked with 13-C, at 45 days after finalising the treatment. A minimum of 15 days of suspension of any proton pump inhibitor was required, and a similar period of abstinence from antibiotics before the UBT. The test was carried out by personnel who were unaware of the medication taken by the patient, using the infrared spectrophotometry procedure, which has a diagnostic efficacy similar to that obtained by mass spectrophotometry [22,23] (UbiT -IR 300 - Otsuka Electronics Co., Ltd). The results were considered to be negative when the ratio obtained between the baseline sample and that obtained after the administration of urea marked with $13-\mathrm{C}$ was $\leq 2.50 / 00$ and positive if this value was higher than $2.50 / 00$. The analysis of efficacy was planned: a) by intention to treat (ITT), on all the patients included in the trial (in the case of loss of follow up, this was considered a positive result in the experimental group and a negative one in the quadruple therapy following the worse case method); b) per protocol (PP), which included only the patients who had completed the whole follow up procedure and had consumed at least $90 \%$ of the medication provided.

\section{Secondary variable}

Adverse side effects were recorded on a purpose-made form, at 7 and 45 days after finalising the treatment. Such effects were considered mild if they were well tolerated by the patient, moderate if they were of sufficient intensity as to interfere with the patient's normal life or as to require medical intervention, serious when they impeded the performance of everyday activities, significantly affected the clinical situation and justified medical intervention, and acute when the patient's life was put at risk.

\section{Sample size}

The sample size was calculated according to a forecast eradication rate of $67 \%$ with the QT (based on the values obtained in preliminary studies at our Hospital, unpublished data) and on a forecast success rate of $84 \%$ for OAR, with a level of significance of 0.05 and a power of 0.80 . We foresaw a minimum number of 102 patients per group, this figure including a $10 \%$ oversizing element. The trial was designed in the expectation of an intermediate analysis when half of the calculated sample size had been achieved; the trial would be interrupted if a difference equal to or greater than $30 \%$ determined by ITT was found, if such a difference were statistically significant.

\section{Statistical analysis}

A descriptive statistical analysis was carried out, with measures of central trend and dispersion for the continuous variables, and frequency distribution for the qualitative variables, comparing the baseline levels of the two groups of patients for the membership variables (age and sex) and clinical variables (type of endoscopic injury, tobacco consumption, defined as smoking 10 or more cigarettes per day). We compared the rate of eradication in each treatment group, using the odds ratio (OR) of incidence, and the chi squared test was used against the hypothesis. A multivariate logistic regression model was built adjusting for basal variables. The level of statistical significance was established as $\mathrm{p}<0.05$.

\section{Results}

Patients were included in the study from September 2004 until August 2005, and an intermediate analysis was performed (from which definitive results were obtained) when half of the initially scheduled sample had been examined. Figure 1 shows that of a total of 102 patients, three were excluded.

Of the 99 patients definitively included, 45 were assigned to Group A (OAR) and 54 to Group B (QT). Table 1 shows that the groups were homogeneous with regard to sex, age, tobacco consumption and endoscopic injury. Although there was a higher proportion of ulcerous patients in the QT group, this difference was not statistically significant. 


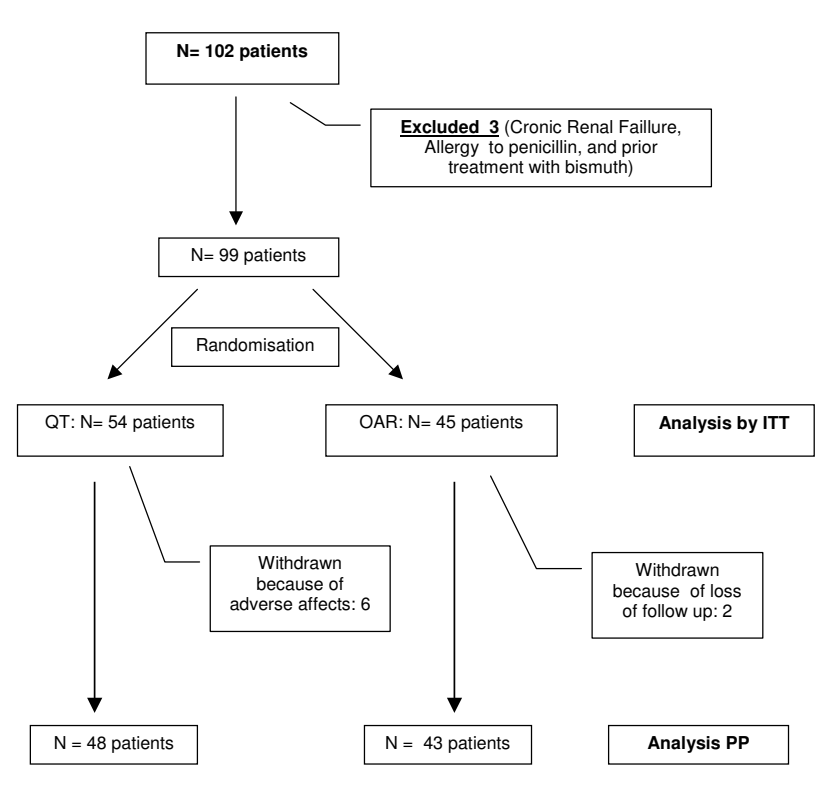

Figure I

Inclusion and flow of patients. ITT: Intention to Treat. PP: Per Protocol.

The QT group presented a higher consumption of NSAIDs.

Table 2 illustrates the efficacy of the two treatments. By intention to treat (ITT), in the QT group, HP was eradicated in 38 of the 54 patients $(70.4 \%)$, while the OAR treatment only eradicated it in 20 of the 45 cases $(44.4 \%)$; the difference was statistically significant $(\mathrm{p}=0.009$; OR = 1.58). In the per protocol (PP) analysis, similar differences were observed: a cure was achieved in 37 of the 48 patients who completed the treatment (77\%), and in 20 of the 43 who finalised the OAR programme (45\%); this difference was also statistically significant $(p=0.002)$. One patient in the QT group achieved HP eradication despite not having completed the course of treatment. The adjustment for the basal variables in the model of logistic regression did not modify these results (data not shown).

We analysed the efficacy achieved with respect to the endoscopic injury and no differences were found in eradication rates between ulcerous (56.8\% of 44 cases) and nonulcerous patients (57.7\%).

The treatment was not completed in 8 cases: 6 in the QT group because of unacceptable side effects (UBT control was achieved in all), and 2 in the OAR group, because of loss of follow up.

Table 3 shows the adverse events recorded. In the QT group 35 patients (64\%) reported at least one adverse event, versus $44 \%$ of the patients in the OAR group ( $\mathrm{p}=$ 0.042 ). The most commonly described such events were epigastralgia and/or dyspepsia, taste loss or variation, dizziness, diarrhoea, nausea and/or vomiting, loss of appetite and cephalea. Severe effects were much less frequent. No leucopoenia was found. 14 events were classified as severe; of these, 11 were in the QT group and 3 in the OAR group. No patient in the latter group stopped medication for this reason.

\section{Discussion}

A controlled study was carried out to compare a quadruple therapy and an experimental one with rifabutin (associated with omeprazole and amoxicillin), as second-line treatment. The efficacy obtained with the rifabutin regimen was 44\% (ITT) and 45\% (PP), greatly inferior to that obtained with the quadruple therapy $(70 \%$ and $76 \%$, respectively).

HP infection is not easy to eradicate when the first-line treatment fails. The most important predictors of failure are antibiotics resistance and regimen compliance. Quadruple therapy has an estimated efficacy of about 75\% [24],

Table I: Demographic data of the patients.

\begin{tabular}{|c|c|c|c|}
\hline & $\mathrm{QT}(\mathrm{n}=54)$ & $\operatorname{OAR}(n=45)$ & $P$ value \\
\hline Age & 46.4 & 48.6 & ns \\
\hline Sex (male/female) & $25 / 29$ & $23 / 22$ & ns \\
\hline Caucasian & $96.3 \%$ & $97.7 \%$ & \\
\hline \multicolumn{4}{|l|}{ Endoscopic findings } \\
\hline Chronic gastritis & 25 (46.3\%) & $28(62.2 \%)$ & \\
\hline GD Ulcer & $29(53.7 \%)$ & $17(37.8 \%)$ & ns \\
\hline Smokers & $12(22.2 \%)$ & $10(22.2 \%)$ & ns \\
\hline Alcohol consumers & $15(27.8 \%)$ & I I (24.4\%) & ns \\
\hline ASA/NSAID & $10(18.5 \%)$ & $2(4.4 \%)$ & 0.031 \\
\hline
\end{tabular}

GD Ulcer: Gastroduodenal ulcer Smokers $\geq 10$ cigarettes per day Alcohol consumers $\geq 2$ units per day ns: no significant 
Table 2: Eradication rates of the study population.

\begin{tabular}{|c|c|c|c|c|c|c|}
\hline \multirow[b]{2}{*}{ UBT } & \multicolumn{3}{|c|}{ Intention to Treat } & \multicolumn{3}{|c|}{ Per Protocol } \\
\hline & QT & OAR & $\mathrm{Cl} 95 \%$ & QT & OAR & $\mathrm{Cl} 95 \%$ \\
\hline Negative & 38 (70.4\%) & $20(40.4 \%)$ & $p=0.009$ & 37 (77.1\%) & $20(46.5 \%)$ & $p=0.003$ \\
\hline Positive & $16(29.6 \%)$ & $25(55.6 \%)$ & & II (22.9\%) & $23(53.5 \%)$ & \\
\hline$N$ & 54 & 45 & OR I.58 (1.I-2.29) & $48 *$ & 43 & OR I.65 (1.1-2.36) \\
\hline
\end{tabular}

* One patient achieved eradication without having completed the treatment programme

but is uncomfortable to administer, because of the large number of pills required; moreover, it provokes side effects that have a negative influence on completion of the regimen, in normal clinical practice [8]. For these reasons, many attempts have been made to find alternative treatment regimens that do not present the above problems. Most such options are based on alternative antibiotics, because the repetition of treatment cycles based on the same antibiotics, especially clarithromycin and/or metronidazole, has no possibility whatever of success [25].

Table 3: Side effects in each treatment group.

\begin{tabular}{|c|c|c|}
\hline & $Q T$ & $O A R$ \\
\hline Total patients & $35(64 \%) *$ & $20(44 \%) *$ \\
\hline \multicolumn{3}{|l|}{ Dyspepsia } \\
\hline+ & 17 & 8 \\
\hline+++ & 1 & 1 \\
\hline \multicolumn{3}{|c|}{ Taste-sensation alteration } \\
\hline+ & 12 & 5 \\
\hline+++ & 2 & \\
\hline \multicolumn{3}{|l|}{ Dizziness } \\
\hline+ & 13 & 4 \\
\hline+++ & 1 & \\
\hline \multicolumn{3}{|l|}{ Diarrhoea } \\
\hline+ & 10 & 6 \\
\hline+++ & 1 & \\
\hline \multicolumn{3}{|l|}{ Nausea/Vomiting } \\
\hline+ & 10 & 5 \\
\hline+++ & 3 & 1 \\
\hline \multicolumn{3}{|l|}{ Loss of appetite } \\
\hline+ & 9 & 6 \\
\hline+++ & 2 & \\
\hline \multicolumn{3}{|l|}{ Cephalea } \\
\hline+ & 8 & 6 \\
\hline+++ & 1 & 1 \\
\hline \multicolumn{3}{|l|}{ Odynophagia } \\
\hline+ & 4 ( 2 candidiasis) & \\
\hline \multicolumn{3}{|l|}{+++} \\
\hline \multicolumn{3}{|l|}{ Asthenia/tiredness } \\
\hline+ & 2 & 3 \\
\hline \multicolumn{3}{|l|}{+++} \\
\hline Total episodes & 85 & 43 \\
\hline Total episodes +++ & 11 & 3 \\
\hline
\end{tabular}

$$
\begin{aligned}
& * \text { p } 0.042 \\
& +:: \text { mild/moderate } \\
& +++: \text { : severe }
\end{aligned}
$$

In preliminary studies, rifabutin-based therapy had good results in patients with one or more failed attempts at eradication, both for patients who are sensitive to antibiotics and for those who have developed secondary resistance to clarithromycin and metronidazole as a result of previous treatments $[16,26]$. In such studies, the antibiotic association most often used is rifabutin $300 \mathrm{mg} /$ day and amoxicillin ( $1 \mathrm{gr} / 12 \mathrm{~h})$, although tests have also been made with levofloxacin [27].

The present study describes the first clinical trial designed to compare a rifabutin-based treatment, as a second-line rescue treatment, with quadruple therapy, which is the standard recommended. The previous results obtained with rifabutin-based therapy had been very promising, with eradication rates of $82-91 \%[10,16,19,26,27]$. However, our clinical study does not corroborate the above eradication rates and was found to be less effective than the quadruple therapy. The latter, on the other hand, in our series achieved a success rate similar to that found by other authors [24].

The eradication rate obtained with our rifabutin-based regimen is one of the lowest published to date. In the first trial reported, Perri et al. [16] obtained an effectiveness of $80 \%$ with a regimen similar to ours, surpassing the $66 \%$ obtained with the quadruple therapy. Perhaps the different duration of the treatment implemented (10 days) may partially explain the difference in the results achieved. Other authors, however, have published similar results with rifabutin-based therapy for seven days: Bock [10] reported an efficacy of $71 \%$ in a non-comparative prospective study, in combination with amoxicillin and pantoprazole. Longer treatment periods, nevertheless, are not reported to improve success rates: Gisbert [19] obtained an eradication rate of $79 \%$ in a series limited to 14 cases, as a third-line rescue treatment, in which the regimen was administered for two weeks. Torachio [26] published a study of a 10 day regimen with amoxicillin and pantoprazole, applied only to patients with resistance to clarithromycin and metronidazole (whether primary or induced by previous eradication treatment). An eradication rate of $87 \%$ was achieved among the patients who had not been treated previously and one of $78.5 \%$ among those with a history of failed prior eradication. Recently Borody [28] 
has reported an eradication rate of $96.6 \%$ with a lower dose of rifabutin (150 mg per day) and an increase in the dose of amoxicillyn (1.5 gr. tid) and PPI's (pantoprazole $80 \mathrm{mg}$ tid) for 12 days.

Associated with antibiotics other than amoxicillin, Wong [27] published a comparative study with quadruple therapy applied to patients with one or more previous attempts at eradication, associating rifabutin $(300 \mathrm{mg}$ day) with levofloxacin (500 mg day); this also gave good results (91\%, versus $90 \%$ with the quadruple therapy). In this line, levofloxacin-based triple therapy is a novel and promising alternative that seems to offer advantages over quadruple therapy [29].

To date, only two studies have described an eradication rate comparable to ours. Quasim [30], in a series of 34 patients included prospectively after two failures, obtained eradication in only $38 \%$ of the cases, and the second one, reported by Gisbert (31), achieved -with a rifabutin-based third line therapy- only an eradication rate of $44 \%$. These studies, together with ours, are the only ones to obtain such a disappointing outcome.

Therefore, we are faced with the first clinical trial to obtain unsatisfactory results using a rifabutin-based therapy as second line treatment. We can find no reasonable explanation for the results obtained. In the randomisation of our patients, the group treated with the quadruple therapy included more ulcerous patients than did the rifabutin group. Patients with a gastroduodenal ulcer seemed to respond better to the eradication treatment than did those with chronic gastritis [32]. This might be an explanation but our patients with a gastroduodenal ulcer had a response rate $(56.8 \%)$ similar to that of the non-ulcerous ones $(57.7 \%)$, and so this bias in the randomisation does not seem to have influenced the results obtained.

A priori, nor should the use of amoxicillin in this regimen have had a negative impact, as a failed eradication treatment does not increase the risk of secondary resistance to this antibiotic. Not having performed an antibiogram study before inclusion of the patients might represent a limitation of the present study, but it seems to have been proved that resistance to clarithromycin and metronidazole does not influence the effectiveness of rifabutinbased treatments $[16,26,27]$, and primary resistance to rifabutin is minimal. Moreover, amoxicillin presents a very low rate of primary resistance in our geographical area [33].

One possible explanation for our results, compared with those of other authors, would be the duration of the treatment. Perhaps 7 days is not sufficient for a rifabutin-based therapy. Nevertheless, our study was designed for a simi- lar duration in both groups, and guidelines recommended quadruple-based therapy for seven days [1]. Other possible explanations might be related to differences obtained in different geographical areas, as recently reported by Gisbert [31] in a study conducted in Spain.

The most plausible explanation, nevertheless, would be the amount of drug dose that was administered. As Borody reported recently [28], a course of higher doses of amoxicillin (1 gr or $1.5 \mathrm{gr}$ tid) and pantoprazole ( $80 \mathrm{mg}$ tid) for 12 days, even with low dose of rifabutin $(150 \mathrm{mg}$ daily), was well-tolerated and proved to be highly effective as a rescue therapy for patients having failed previous treatment. Therefore standard dose of amoxicillin (1gr bid) and PPI's (omeprazole $20 \mathrm{mg}$ bid) might not be sufficient as a rescue therapy, and a better dosage should be sought in future comparative studies.

As is the case in other series that have been described, we found that quadruple therapy frequently provokes side effects that produce a reduction in the fulfilment of the therapy $(11 \%$ of the patients included in the quadruple therapy regimen did not complete the recommended programme). Of the total of 14 events classified as serious or acute, most of them (11 events) corresponded to the quadruple therapy regimen. The rifabutin treatment, on the other hand, was well tolerated, and its side effects were mainly classified as mild, and had no consequences on the final rate of fulfilment of the treatment programme.

\section{Conclusion}

In summary, in the present study a rifabutin-based therapy, associated to standard dose of amoxicillin and omeprazole applied for 7 days was not found to be effective as a second line rescue treatment against HP infection. The difficulty in administration and the frequency of side effects associated with quadruple therapy treatment underline the need to search for alternative options, with a different dose of rifabutin-based triple therapy or with alternative antibiotics which should be better tolerated, easier to administer and which match or improve upon the effectiveness achieved with this reference regimen.

\section{Competing interests}

The author(s) declare that they have no competing interests.

\section{Authors' contributions}

All authors contributed to the design of the study. Revision of the different versions of the study protocol: JMN, NF, FLS, EC, MC, LMR, EPM. Substantial contributions to the conception and design of the digital data record: JMN, NF, FLS, EC, MC, LMR, RR, EU, FV, IM, EPM. Acquisition of data and quality control: JMN, NF, FLS, EC, MC, LMR, RR, EU, FV, IM, EPM, JLM. Analysis and interpretation of 
data: JMN, EPM, FRR. All authors have read and approved the final manuscript.

\section{Acknowledgements}

To Sociedad Andaluza de Patología Digestiva for promoting this trial.

To Pfizer España for supplying, free of charge, the rifabutin capsules necessary for the study.

To Inma Martin and Sensi Guerrero, our nursing assistants, for their dedication and skill.

To the technical staff of the pharmaceutical service of Hospital Costa del Sol, for the patience shown.

To everybody who, in one way or another, contributed to the successful performance of this project.

\section{References}

I. Malfertheiner P, Megraud F, O'Morain C, Hungin AP, Jones R, Axon A Graham DY, Tytgat G, European Helicobacter Pylori Study Group (EHPSG): Current concepts in the management of Helicobacter pylori infection - the Maastricht 2-2000 Consensus Report. Aliment Pharmacol Ther 2002, I6:167-80.

2. Lam SK, Talley NJ: Report of the 1997 Asia Pacific Consensus Conference on the management of Helicobacter pylori infec tion. J Gastroenterol Hepatol 1998, I3: I-I2.

3. Huang JQ, Hunt RH: Treatment after failure: the problem of "non-responders". Gut 1999, 45:140-4.

4. Lee JM, Breslin NP, Hyde DK, Buckley MJ, O'Morain CA: Treatment options for Helicobacter pylori infection when proton pump inhibitor-based triple therapy fails in clinical practice. Aliment Pharmacol Ther 1999, 13:489-96.

5. Gisbert JP, Gisbert JL, Marcos S, Gravalos RG, Carpio D, Pajares JM Seven-day 'rescue' therapy after Helicobacter pylori treatment failure: omeprazole, bismuth, tetracycline and metronidazole vs. ranitidine bismuth citrate, tetracycline and metronidazole. Aliment Pharmacol Ther 1999, 13:1311-6.

6. Boixeda D, Bermejo F, Martin-De-Argila C, Lopez-Sanroman A Defarges V, Hernandez-Ranz F, Milicua JM, Garcia-Plaza A: Efficacy of quadruple therapy with pantoprazole, bismuth, tetracycline and metronidazole as rescue treatment for Helicobacter pylori infection. Aliment Pharmacol Ther 2002, I 6: |457-60.

7. Michopoulos S, Tsibouris P, Bouzakis H, Balta A, Vougadiotis J, Broutet $\mathrm{N}$, Kralios N: Randomized study comparing omeprazole with ranitidine as anti-secretory agents combined in quadruple second-line Helicobacter pylori eradication regimens. Aliment Pharmacol Ther 2000, 14:737-44.

8. Katelaris PH, Forbes GM, Talley NJ, Crotty B: A randomized comparison of quadruple and triple therapies for Helicobacter pylori eradication: The QUADRATE Study. Gastroenterology 2002, 1 23:1763-9.

9. Perri F, Festa $V$, Andriulli A: Treatment of antibiotic-resistant Helicobacter pylori. N Engl J Med 1998, 339:53.

10. Bock H, Koop H, Lehn N, Heep M: Rifabutin-based triple therapy after failure of Helicobacter pylori eradication treatment: preliminary experience. / Clin Gastroenterol 2000, 3 I :222-5.

II. Heep M, Beck D, Bayerdorffer E, Lehn N: Rifampin and rifabutin resistance mechanism in Helicobacter pylori. Antimicrob Agents Chemother 1999, 43:1497-9.

12. Akada JK, Shirai M, Fujii K, Okita K, Nakazawa T: In vitro anti-Helicobacter pylori activities of new rifamycin derivatives, KRM1648 and KRM-1657. Antimicrob Agents Chemother 1999, 43:1072-6.

13. Brogden RN, Fitton A: Rifabutin. A review of its antimicrobial activity, pharmacokinetic properties and therapeutic efficacy. Drugs 1994, 47:983-1009.

14. Rossi $R$, Jabes D, Della Bruna C: In vitro activity of rifabutin, potential antibiotic in the therapy of Helicobacter pylori. Abstract Book of the 6th International Congress for Infectious Diseases, Prague (Czech Republic) 1994:69.

15. Rossi G: [An update on the antibiotic therapy of tuberculosis] Recenti Prog Med 1999, 90:24I-3.

16. Perri F, Festa V, Clemente R, Villani MR, Quitadamo M, Caruso N, Bergoli ML, Andriulli A: Randomized study of two "rescue" thera- pies for Helicobacter pylori-infected patients after failure of standard triple therapies. Am / Gastroenterol 2001, 96:58-62.

17. Griffith DE, Brown BA, Girard WM, Wallace RJ Jr: Adverse events associated with high-dose rifabutin in macrolide-containing regimens for the treatment of Mycobacterium avium complex lung disease. Clin Infect Dis 1995, 2 I:594-8.

18. Pilotto A, Franceschi M, Rassu M, Furlan F, Scagnelli M: In vitro activity of rifabutin against strains of Helicobacter pylori resistant to metronidazole and clarithromycin. Am J Gastroenterol 2000 , 95:833-4.

19. Gisbert JP, Calvet X, Bujanda L, Marcos S, Gisbert JL, Pajares JM: 'Rescue' therapy with rifabutin after multiple Helicobacter pylori treatment failures. Helicobacter 2003, 8:90-4.

20. Canducci F, Ojetti V, Pola P, Gasbarrini G, Gasbarrini A: Rifabutinbased Helicobacter pylori eradication 'rescue therapy'. Aliment Pharmacol Ther 200I, I 5:| 43.

21. de Boer WA, Thys JC, Borody TJ, Graham DY, O'Morain C, Tytgat GN: Proposal for use of a standard side effect scoring system in studies exploring Helicobacter pylori treatment regimens. Eur I Gastroenterol Hepatol 1996, 8:64l-3.

22. Koletzko S, Haisch M, Seeboth I, Braden B, Hengels K, Koletzko B, Hering P: Isotope-selective non-dispersive infrared spectrometry for detection of Helicobacter pylori infection with I3Curea breath test. Lancet $1995,345: 96 \mid-2$

23. Gisbert JP, Gomollon F, Dominguez-Munoz JE, Borda F, Jimenez I, Vazquez MA, Gallego S, Iglesias ], Pastor G, Pajares JM: [Comparison between two I3C-urea breath tests for the diagnosis of Helicobacter pylori infection: isotope ratio mass spectrometer versus infrared spectrometer]. Gastroenterol Hepatol 2003, 26: $14 \mid-6$.

24. Hojo M, Miwa $\mathrm{H}$, Nagahara A, Sato N: Pooled analysis on the efficacy of the second-line treatment regimens for Helicobacter pylori infection. Scand J Gastroenterol 200I, 36:690-700.

25. de Boer WA, Tytgat GN: Regular review: treatment of Helicobacter pylori infection. BM/ 2000, 320:3।-4

26. Toracchio S, Capodicasa S, Soraja DB, Cellini L, Marzio L: Rifabutin based triple therapy for eradication of $\mathrm{H}$. pylori primary and secondary resistant to tinidazole and clarithromycin. Dig Liver Dis 2005, 37:33-8.

27. Wong WM, Gu Q, Lam SK, Fung FM, Lai KC, Hu WH, Yee YK, Chan CK, Xia HH, Yuen MF, Wong BC: Randomized controlled study of rabeprazole, levofloxacin and rifabutin triple therapy vs. quadruple therapy as second-line treatment for Helicobacter pylori infection. Aliment Pharmacol Ther 2003, I 7:553-60.

28. Borody TJ, Pang G, Wettstein AR, Clancy R, Herdman K, Surace R, Lorente R: Efficacy and safety of rifabutin-containing "rescue therapy " for resistant Helicobacter pulori infection. Aliment Pharmacol Ther 2006, 23(4):48I-8.

29. Saad RJ, Schoenfeld P, Kim HM, Chey WD: Levofloxacin-based triple therapy versus bismuth-based quadruple therapy for persistent Helicobacter pylori infection: a meta-analysis. Am J Gastroenterol 2006, I 01 :488-96

30. Qasim A, Sebastian S, Thornton O, Dobson M, McLoughlin R, Buckley $\mathrm{M}$, O'Connor $\mathrm{H}$, O'Morain $\mathrm{C}$ : Rifabutin- and furazolidone-based Helicobacter pylori eradication therapies after failure of standard first- and second-line eradication attempts in dyspepsia patients. Aliment Pharmacol Ther 2005, 2 I:91-6.

31. Gisbert JP, Gisbert JL, Marcos S, Moreno-Otero R, Pajares JM: Thirdline rescue therapy with levofloxacin is more effective than rifabutin rescue regimen after two Helicobacter pylori treatment failures. Aliment Pharmacol Ther 2006, 24(10):1469-74

32. Gisbert JP, Marcos S, Gisbert JL, Pajares JM: Helicobacter pylori eradication therapy is more effective in peptic ulcer than in non-ulcer dyspepsia. Eur J Gastroenterol Hepatol 200 I, I 3: I 303-7.

33. Montiel N, Navarro JM: Primary antibiotics susceptibility of Helicobacter pylori in a general hospital. I4th European Congress of clinical and microbiology and infectious diseases. Prague 2004, Abstracts P742: 184 .

\section{Pre-publication history}

The pre-publication history for this paper can be accessed here:

\section{http://www.biomedcentral.com/1471-230X/7/31/pre}

pub 